\title{
Indicadores para Avaliação da Formação e Aproveitamento Vocacional em Ciências Exatas: Um Estudo de Caso
}

Indicators for the Evaluation of the Quality of Education and Career Development in the Hard Science: a Case Report

\author{
Lilia I. Arany-Prado \\ Universidade Federal do Rio de Janeiro \\ Observatório do Valongo \\ Lad. Pedro Antônio 43 \\ 20080-090 Rio de Janeiro, RJ, Brazil \\ lilia@ov.ufrj.br
}

Recebido em 29 de novembro, 2002. Aceito em 18 de fevereiro, 2003.

\begin{abstract}
São apresentados resultados de uma auto-avaliação abrangente do curso de graduação de Astronomia do Observatório do Valongo da Universidade Federal do Rio de Janeiro. Foram criados indicadores capazes de quantificar os reflexos dos aperfeiçoamentos curricular e institucional sobre a formação e aproveitamento vocacional ao longo de 30 anos. É notável nesse estudo que a instituição caracteriza-se por: i) seu rápido desenvolvimento recente; ii) ter a graduação como um investimento de longa duração e possuir uma base de dados quase completa; iii) estar inserida em uma sociedade jovem (Sociedade Astronômica Brasileira), com alta taxa de crescimento. Isso permitiu diagnosticar a trajetória institucional e a adequação da estrutura do curso às diferentes necessidades da carreira. Mostra-se que, tratando-se de ciência exata, o aumento dos percentuais de alunos formados e a diminuição do tempo permanência na graduação não são suficientes, ou mesmo adequados, para avaliar a eficiência do ensino.
\end{abstract}

\begin{abstract}
We report the results of the self-evaluation of the Astronomy Bachelor Course of the Observatório do Valongo, Universidade Federal do Rio de Janeiro. We have created data indicators capable of quantifying the impact of curriculum and institutional improvements on the student's background and career development in the last 30 years. It is remarkable that the institution in study: i) has undergone appreciable recent development; ii) has its graduation course as a long term investment and an essentially complete database on past professors and students; iii) is inserted in a young scientific Society (Sociedade Astronômica Brasileira), which has shown marked recent maturation and a large rate of growth. This enabled us to diagnose the evolution of the institution and its suitability to the different requirements of the astronomical career. We show that, in the hard science field, the increase of the number of graduated students and the decrease of the time spent as an undergraduate student are not sufficient, or even adequate, to evaluate the efficiency of education in science.
\end{abstract}

\section{Introdução e Histórico}

Esse trabalho originou-se na auto-avaliação do curso de graduação do Observatório do Valongo (OV)/UFRJ que oferece o título de Astrônomo. A avaliação subsidiou parcialmente as discussões sobre a nova reforma curricular em vias de ser formalmente implantada e esteve inserida, também, na avaliação global da Universidade proposta por uma comissão da UFRJ, em 1995. Tal proposta baseou-se na necessidade de "otimizar a relação entre Universidade e Sociedade", que "vem exigindo maior eficácia das ações de seus órgãos públicos", considerando: a) a escassez de recursos a que um país emergente está submetido e a magnitude dos problemas sociais que é preciso enfrentar; b) que o papel es- sencial da Universidade é a formação de recursos humanos qualificados [1, pag 271].

Em meio às freqüentes discussões sobre a eficiência do ensino de terceiro grau, tem sido sugerido um aumento imediato da razão de aluno formado por professor e a diminuição do tempo de permanência do aluno na Universidade. Entretanto, a formação científica implica um crescimento de complexidade que dificilmente pode alcançar, de forma abrupta, os índices baseados em médias de alunos formados nas diversas profissões em alguns outros países. Particularmente, índices de diplomação são peculiares para a formação científica em ciências exatas. Assim, as razões e os números desejados para avaliação de eficiência não deveriam ser analisados sem algum tipo de índice de aprovei- 
tamento vocacional como por exemplo os apresentados na seção IV desse trabalho, que apontam algumas peculiaridades, como mencionado acima.

$\mathrm{Na}$ tentativa de criar os corretos parâmetros de avaliação do curso de Astronomia, foi utilizada uma metodologia não embasada teoricamente, mas nascida do estudo do caso em si. Chegou-se heuristicamente a indicadores como, por exemplo, os tempos de qualificação [2], resumidos na figura 7 desse trabalho, capazes de quantificar a influência do aprimoramento do ensino sobre a futura qualificação de alunos. Nesse trabalho também é mostrada, através de indicadores de aproveitamento de ex-alunos do curso (figuras 6 e 8), a compatibilidade entre o aprimoramento curricular da graduação e as necessidades do mercado devido ao grande desenvolvimento recente da área no Brasil.

Pode-se assumir a pesquisa realizada como geradora de indicadores alternativos para avaliação de curso, constituindo-se parcialmente suas análises em uma justificativa das soluções "semi-empíricas" encontradas.

O estudo de caso, abrangendo um período tão longo quanto o aqui apresentado, só foi possível porque envolveu um número relativamente pequeno de alunos. Houve dificuldades na obtenção de dados. Por exemplo, não havia registros de fácil acesso para calcular os tempos de qualificação dos dois primeiros intervalos da figura 7; também muitas informações foram obtidas através da busca, utilizando vários meios de comunicação, de ex-alunos no Brasil e no exterior. Ou seja, a falta de recursos computacionais do passado não permitiu o armazenamento de dados, que hoje em dia, realizado de forma eficiente e direcionado a objetivos específicos, tornaria o trabalho relativamente simples. Deve ser ressaltado, entretanto, que o número pequeno de alunos envolvidos na pesquisa não significa um percentual baixo de alunos formados por ano, quando se compara a outros cursos em ciências exatas no país.

A fim de ressaltar o grande crescimento do interesse pela Astronomia, o que pode auxiliar no esclarecimento do contexto no qual insere-se essa pesquisa, segue-se um breve histórico das ofertas de cursos e pesquisas na área para alunos de graduação.

O curso de Astronomia foi criado em 1958, na Faculdade Nacional de Filosofia (FNFi) da antiga Universidade do Brasil [3]. O OV, fundado em 1881, pertencia à antiga Escola Politécnica do Rio de Janeiro. À época, localizava-se no morro de Santo Antônio, ao lado do Convento de mesmo nome. Entre 1924 e 1926 foi realizada a mudança para o Morro da Conceição, na então denominada Chácara do Valongo, devido ao desmonte do Morro de Santo Antônio (dentro do projeto de reurbanização do centro da cidade do Rio de Janeiro). Do final da década de 1930 até 1958 o OV ficou abandonado. As atividades ressurgiram após a criação do curso na FNFi. O OV e o curso foram incorporados à UFRJ, depois da Reforma Universitária de 1967 [2]. Após cerca de 40 anos da criação do curso (e ao longo desse tempo, caracterizando-se como o único curso de graduação de Astronomia no Brasil), diversas Universidades com um quadro de professores doutores em Astronomia e Astrofísica, vin- culados a atividades de pesquisa científica, iniciaram programas de graduação na área.

Atualmente, pode-se contabilizar, em cerca de 10 instituições brasileiras de pesquisa e ensino, o oferecimento de cerca de 60 disciplinas formais em todos os níveis de graduação (desde disciplinas introdutórias até as de nível profissional) e o oferecimento de orientação de trabalhos de iniciação científica na área.

Considerando apenas as disciplinas introdutórias de Astronomia oferecidas em Instituições de Ensino Superior do Brasil, Bretones e Compiani [4] analisaram programas e abordagens de 60 disciplinas introdutórias oferecidas por 54 cursos (somente algumas dessas disciplinas introdutórias estão contidas no total de disciplinas formais citadas acima). Segundo os autores, entre os professores responsáveis pelas disciplinas introdutórias, a formação graduada em Física predomina (cerca de $51 \%$, seguida da formação em Geografia, cerca de 10\%). Entre tais professores, há cerca de $53 \%$ de doutores. O trabalho também aponta o fato de que, como afirmam os autores, cerca de $63 \%$ dos docentes iniciaram tal atividade de ensino aproximadamente dentro dos últimos 6 anos. Exceções para tal "juventude" são as do Instituto de Astronomia, Geofísica e Ciências Atmosféricas da Universidade de São Paulo (IAG/USP, originalmente designado por Instituto Astronômico e Geofísico) e do Departamento de Astronomia do Instituto de Física da Universidade Federal do Rio Grande do Sul (UFRGS) que oferecem disciplinas de conhecimento astronômico geral na graduação para vários cursos, respectivamente, desde 1970 e 1972.

Parece haver uma tendência à ampliação da oferta de "graduações" de Astronomia, com uma formação em Astronomia, Física e Matemática semelhantes, oferecendo no entanto diferentes titulações, sendo a quase totalidade delas em Física, como é o caso do IAG. Seus professores ministram as disciplinas de Astronomia do curso de Física com Habilitação em Astronomia da USP (criada em 1997), oferecida pelo Instituto de Física, e outras disciplinas optativas do curso de Física. Deve-se notar também o crescimento da oferta de iniciação científica na área, por parte de instituições com atividades exclusivas de pós-graduação e pesquisa.

O interesse tardio de grande parte da comunidade astronômica brasileira pela formação graduada em Astronomia deve ser um reflexo da juventude da moderna Astronomia Brasileira. Por exemplo, as primeiras teses de doutorado utilizando dados do Laboratório Nacional de Astrofísica (LNA, Brasópolis, MG, o mais importante sítio de observação do país) são de 1988 [5]. A necessidade de impor inicialmente o aspecto profissional da Sociedade Astronômica Brasileira (SAB), criada em 1974, obrigou a fixar esforços na alta qualificação e na pesquisa [6], enquanto que o OV manteve-se bastante isolado em relação à comunidade incipiente. Entretanto, o OV passou por mudanças importantes nos anos 1990. Houve melhorias: na infraestrutura, na qualificação do quadro de professores (figura 1) e nas atividades de pesquisa e extensão. Tais mudanças 
permitiram o crescimento da interação com a comunidade científica. Ainda, está sendo implantada uma pós-graduação no OV (um caminho natural para o aproveitamento de alunos de alto nível egressos do curso de graduação) que tem a finalidade de caracterizar a Unidade como um pólo de Astronomia na UFRJ.

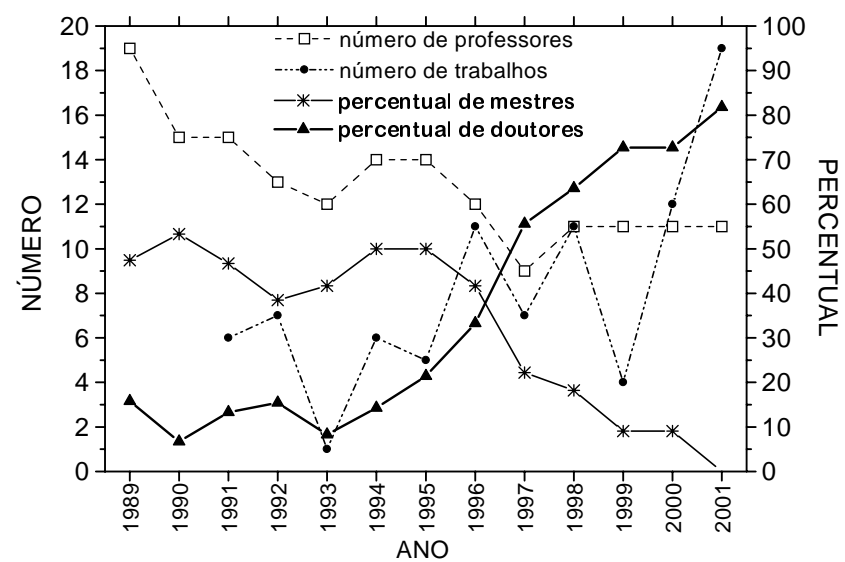

Figura 1. Evolução do quadro, da qualificação e da produção docente no OV: números de professores e de trabalhos (artigos em periódicos e proceedings + teses de doutorado) e percentuais de mestres e de doutores.

A longa duração da atividade de graduação no $\mathrm{OV}$ e o estreito acompanhamento do desenvolvimento discente (a coordenação do curso não tem conhecimento sobre 5 dos 145 alunos formados) permitiram realizar uma ampla avaliação do desempenho de graduados em Astronomia, circunstancialmente a única no gênero no país.

Nesse trabalho é feita um análise atualizada dos levantamentos realizados $[2,7,8]$, acrescentando novos dados e argumentações. Vale ressaltar que a juventude da sociedade (SAB), para a qual tal análise foi inicialmente destinada, aliada a um dos maiores índices de crescimento da área dentre as ciências no Brasil, tornam o trabalho peculiar. Tais circunstâncias amplificam a percepção (em um intervalo de tempo relativamente curto) das influências das melhorias curriculares sobre o aproveitamento vocacional e a qualificação de ex-alunos da graduação.

Uma vez que uma base sólida em Física e Matemática é comum à formação em ciências exatas, espera-se que alguns aspectos dos índices de aproveitamento vocacional apresentados nesse trabalho possam ser estendidos a outros cursos em áreas correlatas.

Na seção II é feito um breve histórico das reformas curriculares e é apresentado o novo currículo, bem como as bases sobre as quais este foi construído. Os indicadores que apontam relações entre os desenvolvimentos implementados no OV e a melhoria na formação graduada são apresentados nas seções III a IV: na seção III descreve-se a evolução do número de alunos formados e da produção discente; na seção IV são introduzidos os principais indicadores gerados pelo estudo de caso, que permitem "medir" o resul- tado da melhoria na formação do graduado sobre sua futura qualificação e sobre o tempo necessário para a qualificação, e também é feita uma análise dos percentuais de contratos profissionais em Astronomia e áreas correlatas. Finalmente, na seção V são feitas algumas observações e conclusões finais.

\section{Formação Graduada e Currículos}

A seguir, é feito um breve resumo da história das reformas curriculares do curso de graduação de Astronomia da UFRJ, apresenta-se a nova reforma que está sendo implantada e descrevem-se as bases sobre as quais esta foi construída.

$\mathrm{Na}$ reforma curricular que se seguiu à Reforma Universitária de 1967, foi introduzido o sistema de créditos e disciplinas semestrais e foi criado um elenco de disciplinas de Astronomia. Logo após, ocorreram alterações nas disciplinas do ciclo básico ministradas pelos Institutos de Física e Matemática e que foram consolidadas em novo currículo, em 1971, tendo sido introduzido então um Projeto Final como requisito complementar para a obtenção do título de Astrônomo. Nova reforma ocorreu em 1975, na qual as principais modificações foram a introdução de disciplinas que tratam de computação, metodologia científica e uma Astronomia/Astrofísica básica ministrada para calouros com o objetivo de fornecer-lhes uma visão geral fenomenológica sobre diversas áreas de atuação. A partir da segunda metade da década de 1970, professores introduziram, em várias disciplinas da Astronomia, adaptações de disciplinas de Física e Matemática. De fato, as reformas vinham acrescentando disciplinas e ementas, resultando um currículo super dimensionado e bastante redundante, que só podia ser cumprido em cinco anos, no mínimo. Isto incentivou uma completa reformulação que resultou na reforma implantada em 1984. Houve redimensionamento em todas as disciplinas e ampliação do conteúdo de Física. O tempo mínimo previsto para a diplomação passou a ser de quatro anos e meio (com a nova reforma curricular, ora em andamento, este tempo será de quatro anos).

A reforma de 1984 foi pautada por um aumento na qualidade da formação, com o objetivo de preparar alunos para ingressarem de forma mais eficiente nas pós-graduações de Astronomia. Tal objetivo foi alcançado e reconhecido na Avaliação Externa pela qual passou a UFRJ. Em [9, pag 109] lê-se: "A partir de 1984, as mudanças curriculares (...) tiveram um papel essencial no aprimoramento da qualidade dos graduados". Ainda, é elogiada a orientação acadêmica: “...a orientação acadêmica (...) tem obtido grande sucesso na adequação do perfil dos novos estudantes e sua progressão no curso". Entretanto, mesmo com uma boa adequação curricular e um acompanhamento acadêmico 
cuidadoso, a tendência de evolução da Astronomia mundial impõe um reforço na formação em Física assim como o aperfeiçoamento de técnicas observacionais que tiveram papel preponderante na construção da identidade da ciência astronômica.

Objetivando atender novas necessidades curriculares, professores do OV estiveram envolvidos nas discussões pertinentes durante anos. Construíram-se diretrizes gerais para a formação, sobre as quais foi estabelecido o perfil desejado do curso, que se segue resumidamente.

Para a formação do astrônomo é fundamental a vivência, treino, análise e síntese, construídos com o enfoque observacional, que o capacite não somente a dominar métodos e técnicas astronômicas, mas que o capacite a pensar no contexto astronômico. Espera-se do astrônomo, que possa trabalhar, por exemplo, com a obtenção e redução de dados de fontes, que são anômalas em relação à realidade subjetiva imediata, as quais estão associadas enormes distâncias, tamanhos e escalas temporais. Espera-se, também, que possa trabalhar com a construção e/ou aplicação de sistemas teóricos e a construção e análise de modelos.

O conteúdo curricular do curso deve: a) ter a flexibilidade necessária para permitir uma constante atualização; b) propiciar ao aluno uma visão ampla da astronomia, compatível com o nível da graduação.

A abordagem dos tópicos deve enfatizar: a) a interpretação da formalização físico-matemática dos temas astronômicos; b) o uso de simulações que permitam a apreensão de conceitos e aspectos teórico-observacionais; c) o uso de modernas técnicas de redução de dados que possibilitem a ligação entre observação e teoria; d) a necessidade de trabalhos extra-classe, como complemento à formação.

Também um currículo, como um sistema complexo organizado, necessita de um componente de ruído sob a forma de uma certa ambigüidade, a que se possa recorrer em caso de crises, para gerar alternativas de soluções adaptativas (sistemas ótimos estão mais sujeitos à destruição). Isto se traduz, por exemplo em algumas regiões de interseção entre disciplinas, na flexibilidade dos conteúdos das disciplinas de especialização e, conforme necessidades específicas dos estudantes, flexibilidade dos pré-requisitos disciplinares.

Entretanto, diante de qualquer flexibilidade na formação do estudante, é fundamental, para a garantia do nível acadêmico, que tal procedimento implique uma estreita orientação acadêmica, envolvendo análises de currículos/históricos e debates com os interessados, objetivando tornar o aluno consciente acerca de sua escolha e o incentivando, conforme sua escolha, a cursar outras disciplinas não obrigatórias e, ainda, a participar de grupos de estudos ou seminários. Tais procedimentos podem frutificar em um acompanhamento "eticamente vivenciado", que consiga receber alunos na tentativa de torná-los produtivos e não meramente julgá-los. Isto pode conduzir, parcialmente, a um meio ambiente interno dinâmico e rico em iniciativa e diversidade, além do oficialmente exigido ou oferecido pelo currículo. Tal riqueza requer, entre outros aspectos, o incentivo ao estabelecimento de grupos de estudo flexíveis, dinâmicos e consequentemente em constante evolução, provocando conectividade entre grupos e indivíduos, alunos e professores. Há, no que foi descrito, um componente fortemente pessoal e espontâneo, que não deve ser tornado oficial (imposto). Associa-se, na verdade, a uma fase de maior complexidade que caracteriza, em parte, uma instituição que busca ser produtiva.

Um exemplo de tal crescimento de complexidade, foi o surgimento de inúmeras atividades não "oficiais", geradas pelo estímulo da discussão e implantação da grande reforma de 1984, que certamente tiveram papel fundamental no crescimento abrupto do número de trabalhos em jornadas de iniciação científica (figura 2, ver também [2]). Outro exemplo, atual, de atividade espontânea é a ajuda oferecida aos calouros, nas disciplinas, por alunos em estágios mais avançados.

A atual reforma aguardava, para sua plena implantação e formalização: as novas resoluções baseadas na Lei de Diretrizes e Bases da Educação Nacional (LDB), a reforma curricular do Instituto de Física e alguns recursos didáticos ora parcialmente disponíveis. Alguns professores no OV têm desenvolvido e participado de projetos de extensão/ensino, com apoio da Fundação Universitária José Bonifácio (FUJB), FAPERJ e Fundação VITAE. Os recursos didáticos provenientes do apoio à extensão também têm sido utilizados para atividades do curso, sem prejuízo das atividades a que se destinam, e servem de incentivo para o aperfeiçoamento e a participação de alunos.

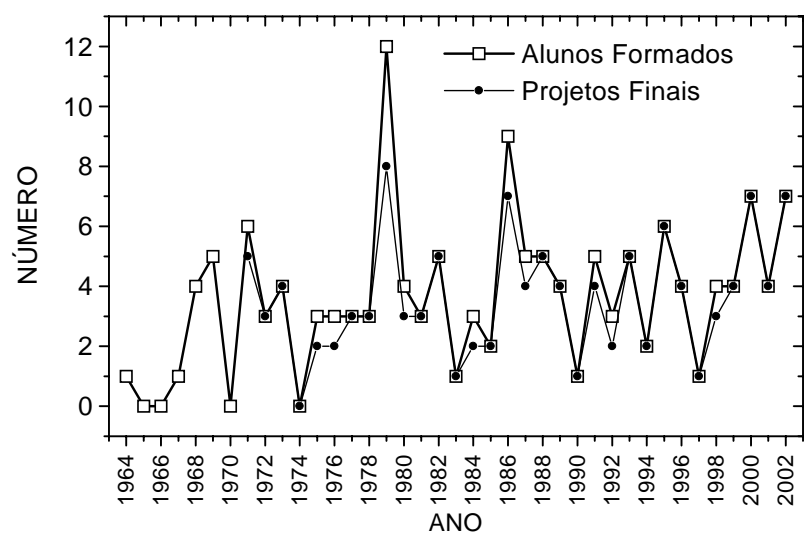

Figura 2. Comparação dos números de alunos formados e de Projetos Finais.

As discussões sobre a nova reforma, se por um lado foram prolongadas por motivos alheios ao OV, levaram entretanto à implementação de várias melhorias curriculares nos últimos anos, que já devem ter reflexos, juntamente com a melhoria da qualificação do quadro de professores (figura 
1), sobre a formação do estudante. Um exemplo é o crescimento recente do número de alunos formados (ver, por exemplo, os números sobre as colunas na figura 5).

Com a implementação da nova reforma, haverá uma diminuição do número total de disciplinas, acompanhada do aumento da carga didática. Esse aumento envolve atividades extra-classe, com ênfase no uso dos recursos computacionais do Laboratório de Informática para a Graduação (LIG). O LIG serve de apoio às aulas de graduação e também funciona como apoio extra-classe na resolução de exercícios, acesso à Web, ferramenta de edição de textos e gráficos, programação, processamento de cálculos e preparação de seminários.

A tabela 1 apresenta um resumo do novo currículo. Encontram-se em negrito as disciplinas ministradas no OV. Os números na tabela referem-se a semestres. O que torna peculiar o currículo do OV, em relação aos cursos que vem sendo implantados em outras instituições, é a opção dada ao estudante de escolher, em seu último ano de formação, entre as duas grandes áreas: Astronomia Dinâmica e de Posição e Astrofísica. Dependendo da escolha, o estudante cursará, de forma concatenada, as Disciplinas Opcionais I e II (tabela 1). Isso torna mais restritivo o conhecimento do graduado nos tópicos mais avançados na grande área descartada por sua escolha. Entretanto, possibilita um aprofundamento, na grande área escolhida, provavelmente maior do que em outras graduações. De fato tal esquema curricular foi bastante discutido no OV e, por fim, optou-se por este, com base em esquema análogo vigente desde a reforma de 1984, e que tem produzido bons profissionais em ambas as grandes áreas.

A reforma visa melhorar a formação dos alunos em Física e em técnicas de observação e de redução de dados em Astronomia. Esse último objetivo será alcançado através da estruturação de disciplinas de caráter eminentemente prático, nas quais serão utilizados dados observacionais obtidos em telescópios no Brasil e no exterior, pelos docentes em seus trabalhos de pesquisa; e também, dentro do possível, a partir de observações nos telescópios disponíveis na Unidade, que foram reformados para atividades didáticas e de extensão, com o apoio da FUJB.

Pretende-se motivar os alunos, iniciando a sua preparação para a participação em projetos observacionais de pesquisa. Essa formação torna-se importante tendo em vista os grandes projetos instrumentais, com vários parceiros internacionais, aos quais está associada a Astronomia Brasileira, que são o GEMINI [10] (ver também [11]) e o Southern Astrophysical Research (SOAR) Telescope (um telescópio de 4,2 metros, instalado em Cerro Pachón, no Chile). O Projeto Gemini consiste de dois telescópios de 8,2 metros: o Gemini Norte em Mauna Kea, no Havaí, e o Gemini Sul, em Cerro Pachón. O SOAR está localizado próximo ao Gemini Sul. Vale ressaltar que foi aprovado em 2001 um Instituto do Milênio (financiado pelo CNPq), coor- denado por Beatriz L. S. Barbuy (IAG/USP), evolvendo 19 instituições brasileiras (o OV incluído), com a finalidade de dotar o SOAR de instrumentação de primeira linha.

\section{Alunos Formados e Produção Dis- cente}

Aqui mostra-se a evolução do número de alunos formados no curso de graduação de Astronomia da UFRJ e a respectiva produção discente: Projetos Finais, trabalhos apresentados nas Jornadas de iniciação científica da UFRJ (iniciadas em 1978) e nas Reuniões anuais da Sociedade Astronômica Brasileira (SAB). Não foi contabilizada a participação de alunos em artigos de revistas indexadas, um fato mais recente e cujo número é relativamente pequeno. $\mathrm{O}$ ano de conclusão de curso, para alunos formados após 1971 (ver seção II), foi assumido como o ano da defesa do Projeto Final. Até 1962, o curso constituía-se em um adendo ao curso de Matemática da FNFi (seção I). Os alunos eram provenientes de instituições militares e nenhum se formou. A primeira formatura é de 1964.

A figura 2 mostra os números de Projetos e de alunos formados por ano. Nota-se na figura que a ocorrência de Projetos conjuntos (com dois ou mais autores) foi mais freqüente no passado. A diminuição de Projetos conjuntos, o que é desejável, indica um maior amadurecimento e diversidade de linhas de pesquisa, com projetos individualizados e melhor estruturados.

Vê-se, ainda na figura 2, um comportamento mais regular e crescente, a partir de 1990. Comparando-se com a figura 1 , nota-se que o início da fase de maior regularidade no número de formados corresponde ao início da queda no número de professores (principalmente devido a aposentadorias). Ocorre que tal queda foi acompanhada de uma renovação do quadro e também do aumento da qualificação de docentes. De fato, a década de 1990 caracteriza uma fase de abertura sistemática para a comunidade que, como conseqüência, entre outros fatos, tornou os alunos mais conscientes da profissão e mais bem preparados para exercê-la.

A figura 3 mostra o número acumulado de alunos formados e o da produção discente. O início do crescimento abrupto dos trabalhos em Jornadas de iniciação científica ocorreu após a reforma curricular de 1984 (seção II). Analogamente, as grandes melhorias ocorridas na década de 1990 (seção I) permitiram que alunos aumentassem a interação com a comunidade, que seus projetos tivessem um salto de qualidade, e que tivesse início a apresentação do trabalho discente nas Reuniões da SAB (antes disso, alunos de graduação não participavam usualmente das Reuniões). O ano de início das publicações dos resumos das Reuniões no Boletim da SAB é 1977. Não houve Reunião da SAB nem Jornada em 1991. 
Tabela 1. Resumo do novo currículo do curso de graduação do OV/UFRJ

\begin{tabular}{|c|c|c|}
\hline $\begin{array}{l}\text { Física I } \\
\text { Física Experimental I } \\
\text { Cálculo Diferencial e Integral I } \\
\text { Introdução à Astronomia Moderna }\end{array}$ & $\begin{array}{l}\text { Física II } \\
\text { Física Experimental II } \\
\text { Cálculo Diferencial e Integral II } \\
\text { Álgebra Linear II } \\
\text { Astronomia Fundamental } \\
\end{array}$ & 2 \\
\hline $\begin{array}{l}\text { Física III } \\
\text { Física Experimental III } \\
\text { Cálculo Diferencial e Integral III } \\
\text { Computação Astronômica } \\
\text { Fundamentos de Técnica Observacional } \\
\end{array}$ & $\begin{array}{l}\text { Física IV } \\
\text { Física Experimental IV } \\
\text { Mecânica Clássica I } \\
\text { Métodos de Física Teórica I } \\
\text { Astronomia Estatística }\end{array}$ & 4 \\
\hline $\begin{array}{l}\text { Eletromagnetismo I } \\
\text { Mecânica Clássica II } \\
\text { Métodos de Física Teórica II } \\
\text { Astrofísica Básica } \\
\text { Fundamentos Teóricos da Astrofísica }\end{array}$ & $\begin{array}{l}\text { Eletromagnetismo II } \\
\text { Termodinâmica e Física Estatística } \\
\text { Astronomia Dinâmica } \\
\text { Astrofísica Estelar } \\
\text { Astronomia Observacional I } \\
\end{array}$ & 6 \\
\hline $\begin{array}{l}\text { Mecânica Quântica I } \\
\text { Física Atômica, Molecular e Óptica } \\
\text { Astrofísica de Plasma } \\
\text { Disciplina Opcional I I }\end{array}$ & $\begin{array}{l}\text { Astronomia Observacional II } \\
\text { Disciplina Opcional II } \\
\text { Projeto Final } \\
\end{array}$ & 8 \\
\hline
\end{tabular}

† Astronomia Dinâmica e de Posição ou Astrofísica Galática e Extragalática

† tópicos avançados em Astronomia Dinâmica e de Posição ou em Astrofísica

Os percentuais de alunos formados pelo curso do OV que apresentaram trabalhos em Jornadas da UFRJ ou em Reuniões da SAB no intervalo 1984-1999 (um total de 233 trabalhos) foram, respectivamente, $78 \%$ e $80,5 \%$. Os percentuais de formação serão maiores se considerarmos os alunos tranferidos que se formaram em outros cursos em áreas correlatas. Isso reforça a constatada relevância da inserção de alunos em projetos de pesquisa ao longo da formação graduada. Segundo Guimarães [12, pag 9], em seus comentários sobre uma avaliação (em 1998) do Programa institucional de bolsas de iniciação científica (Pibic), "a iniciação científica torna praticamente inexistente entre os alunos Pibic a elevada evasão que se observa na graduação"; lê-se ainda: "3 em cada 10 bolsistas chegam ao mestrado e (...) $95 \%$ dos atuais mestrandos, originários do Pibic, pretendem seguir o doutorado". No caso do OV, no intervalo 1984-1999, 5 em cada 10 estudantes que apresentaram trabalhos em congressos (bolsistas ou não) chegaram ao mestrado. Destes, mais do que $50 \%$ já obtiveram o título de mestre e provavelmente haverá cerca de $75 \%$ de doutores (alguns já obtiveram o título).

Na figura 3, diferenças entre o crescimento do número acumulado de alunos formados e o de Projetos Finais, devem-se ao fato de haver Projetos conjuntos ( $c f$. figura 2).

Nas figuras 4 e 5 são mostradas evoluções dos Projetos Finais, em intervalos de oito anos, considerando, respectivamente, algumas áreas da Astronomia (para as quais há projetos em número relevante) e a ênfase de conteúdos em Física ou Matemática. A motivação é comparar alguns aspectos da evolução do interesse da pesquisa discente com a evolução da pesquisa astronômica em geral.

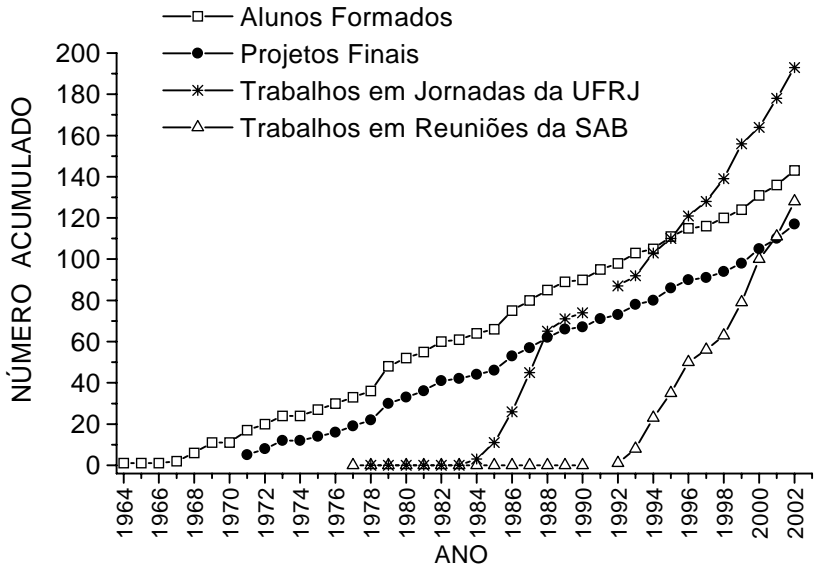

Figura 3. Número acumulado de produção discente, comparado ao número acumulado de alunos formados.

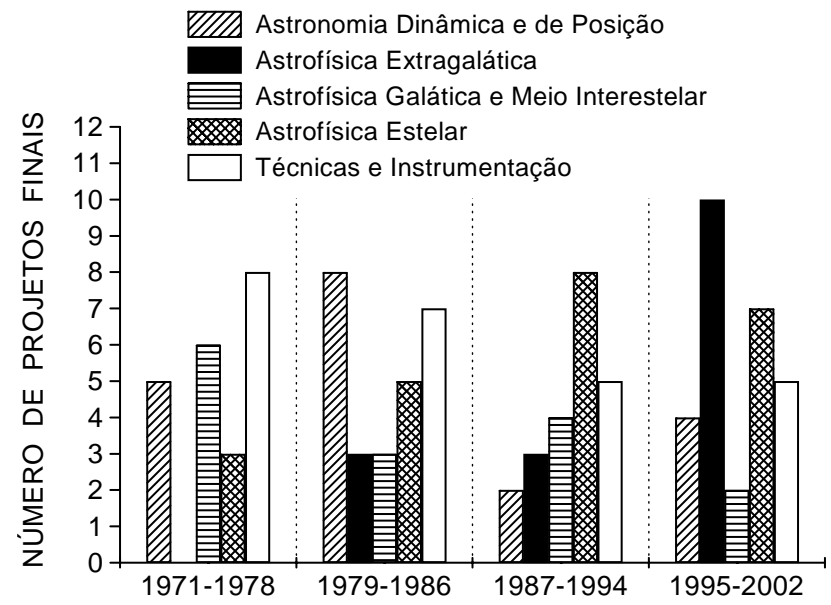

Figura 4. Números parciais de Projetos Finais, em intervalos de oito anos de conclusão de curso, nas áreas que estão representadas por 15 ou mais Projetos Finais no intervalo 1971-2002. 
Na figura 4, as áreas escolhidas para a classificação de projetos seguiram a terminologia utilizada por órgãos de fomento à pesquisa. Na classificação "técnicas e instrumentação", foram incluídos Projetos Finais com conteúdos, por exemplo, de técnicas para tratamento de dados, de métodos matemáticos e softwares, que têm aplicação para diversos objetos astronômicos.

Devido à grande evolução da pesquisa astronômica nos últimos 20 anos no Brasil, a classificação dos Projetos no primeiro intervalo da figura 4 tem um componente subjetivo, pois a classificação aplica-se a um contexto (de menor amadurecimento) muito diverso do atual, principalmente no que se refere à obtenção e redução de dados. Por exemplo, a capacidade de "enxergar" mais longe e melhor, fez crescer o interesse na - hoje denominada informalmente - "cosmologia observacional".

A figura 4 indica duas fases representadas, respectivamente, pelos dois primeiros intervalos e pelos dois últimos, sendo a primeira fase (1971-1986) característica ainda do isolamento do OV, citado na seção I, e a fase mais recente (1987-2002) representando uma evolução de interesses mais condizentes com os da comunidade astronômica. Nota-se ainda que as duas fases estão separadas por alunos anteriores e posteriores à reforma de 1984. O processo de implantação global de uma reforma demanda cerca de 2 anos. Portanto, somente a partir de 1986 o conjunto de todos os alunos do curso estaria sob os requisitos da reforma.

As denominações da Astrofísica, na figura 4, são razoavelmente auto explicativas, no que se refere aos seus objetos de estudo. Uma vez que o público alvo desse trabalho é principalmente de físicos, parece adequado dedicar poucas palavras à Astronomia Dinâmica e de Posição. Esta é uma denominação moderna, mais abrangente, para uma área que inclui segmentos mais tradicionais, como Mecânica Celeste e Astrometria. Particularmente, esta última foi sinônimo de Astronomia até a metade do século dezenove [13]. Durante longo tempo essas foram as únicas frentes de estudo da Astronomia. Entretanto, houve uma expansão do arcabouço teórico da Matemática e da Física, e uma importante evolução das ferramentas computacionais e tecnológicas. Hoje é possível, não somente simular e comparar complexos problemas dinâmicos não lineares com as observações de objetos do Sistema Solar - com aplicações a planetas extrasolares - como também utilizar quasares como sistemas de referência inerciais quasi-ideais.

Segundo Maciel [5], em seu trabalho sobre Astrofísica no Brasil, a Astrofísica Galática e do Meio Interestelar teve sua importância relativa (ao número total de trabalhos em Reuniões da SAB) diminuída, devido parcialmente ao deslocamento de interesse para a área de Astrofísica Extragalática, o que de certa forma está refletido na figura 4.

A figura 5 mostra os percentuais de Projetos Finais com ênfase em Física ou Matemática. Vale observar que tais percentuais não abrangem o total de áreas abordadas nos Projetos. Existem, por exemplo, Projetos em arqueoastronomia, história e filosofia da ciência e ensino (utilizando a Web), não enquadrados nos percentuais da figura 5 .
Nota-se, na figura 5, a queda dos números de Projetos com ênfase em Matemática o que parcialmente justificase pela evolução histórica da Astronomia Dinâmica e de Posição, esboçada acima. Particularmente, o alto percentual inicial com ênfase em Matemática, retrata o isolamento inicial do $\mathrm{OV}$ em relação às atividades da comunidade na áreas da Astrofísica. Além disso, como foi descrito acima, desenvolvimentos recentes tendem a aproximar a Astronomia Dinâmica e de Posição da Astrofísica. Assim, pode-se esperar um renascimento da importância relativa da área e, talvez, um respectivo aumento do número de Projetos, incluindo aí, eventualmente, as linhas com ênfase matemática ( $c f$. figuras 4 e 5). De qualquer forma, o alto percentual de Física na pesquisa discente é um reflexo da principal ferramenta sobre a qual fundamenta-se a grande parte da moderna Astronomia.

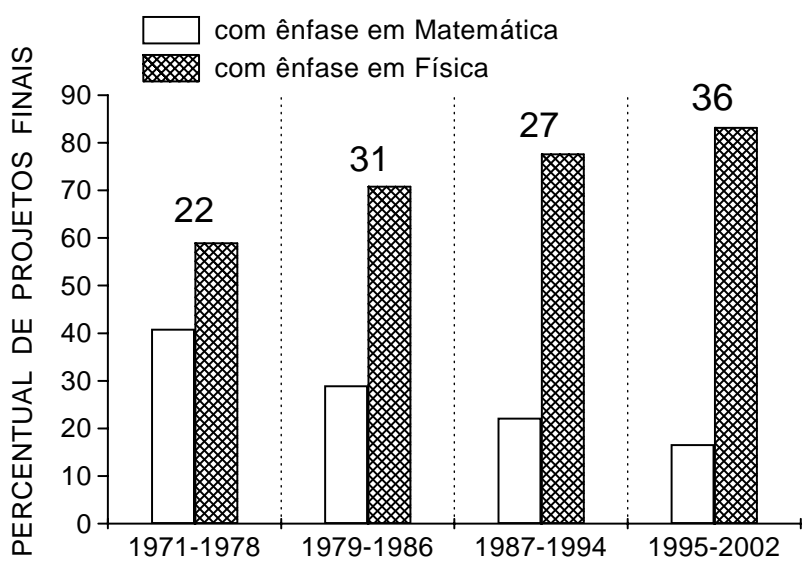

Figura 5. Percentual de Projetos Finais, em relação ao total de Projetos em cada intervalo de oito anos de conclusão de curso, com ênfase em Física ou Matemática. Os números totais de Projetos de cada intervalo estão indicados sobre as colunas.

Ainda vale ressaltar, na figura 5, o grande número relativo de Projetos Finais no último intervalo, o que permite ser otimista quanto ao aumento da média de alunos formados por ano.

\section{Tempos de Qualificação e Apro- veitamento Vocacional de Ex- alunos}

Nesse item mostram-se os principais indicadores, de caráter geral, capazes de retratar, com bastante fidelidade, as influências das melhorias na formação do aluno do curso de graduação de Astronomia da UFRJ. Tais indicadores podem ser tomados como uma prova incontestável do que foi declarado na seção I: que o aumento dos percentuais de alunos formados e que a diminuição do tempo de permanência na graduação (apesar de evidentemente desejáveis) não são su- 
ficientes para avaliar a eficiência do ensino, tratando-se de ciência exata.

$\mathrm{Na}$ figura 6 estão indicados os percentuais de qualificação de mestrado e de doutorado de ex-alunos em Astronomia/Astrofísica ou em Física ou Matemática (em relação aos números de formados a cada sete anos). Os principais institutos que contribuíram para a titulação, de mestre e de doutor, de ex-alunos são: o MCT/Observatório Nacional $(\mathrm{ON})$, o MCT/Instituto Nacional de Pesquisas Espaciais (INPE) e o IAG/USP, para os quais também estão consideradas as qualificações parcialmente realizadas no exterior.

O número inicial significativo de mestres em Física ou Matemática, na figura 6, explica-se em parte pela falta de opções para o aproveitamento vocacional em Astronomia no passado ( $c f$. seção I). No último intervalo da figura, todos os ex-alunos formaram-se sob os requisitos da grande reforma de 1984. É evidente a "explosão" no número de doutores entre tais ex-alunos e é uma prova de que o objetivo dessa reforma foi alcançado (o de melhor preparar o estudante para a pós-graduação em Astronomia/Astrofísica, como descrito na seção II).

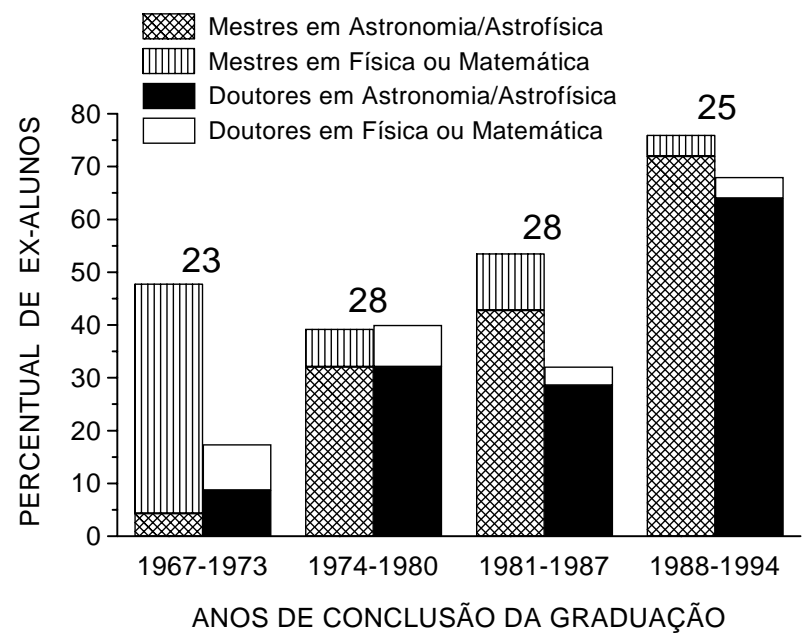

Figura 6. Percentual de alunos que concluíram mestrado e doutorado em relação a alunos que concluíram a graduação. Os dados foram acumulados e os percentuais calculados em intervalos de sete anos de conclusão do curso. O número total de alunos que concluíram a agraduação em cada intervalo de sete anos está indicado sobre as colunas do respectivo intervalo.

A figura 7, que pode se considerada como o melhor índice de eficiência de melhoria na formação do estudante de graduação, mostra a evolução de percentuais de ex-alunos qualificados (graduação, mestrado e doutorado) em menos tempo que algum tempo arbitrado, a partir do ingresso na graduação. Os tempos arbitrados para as qualificações podem ser baseados em alguma fração dos tempos máximos permitidos pelas normas universitárias ou de órgaos de fomento à pesquisa. Os percentuais foram calculados em relação aos números de ingressos (que se formaram) a cada oito anos. No último intervalo da figura, o ponto de interrogação deve-se à estimativa da qualificação de quatro ex-alunos em doutoramento e dois no mestrado, cujas trajetórias acadêmicas permitem prever a conclusão da tese em menos tempo que o arbitrado para a qualificação de doutorado (no último intervalo da figura 7, sete ex-alunos já se qualificaram no critério adotado).

Os intervalos temporais nesse item foram escolhidos de modo a conter a maior quantidade de alunos ou ex-alunos em relação aos quais considerou-se que as informações eram relevantes para as análises. Essa é a razão para os intervalos de oito anos de ingresso na graduação na figura 7 serem diferentes dos intervalos de sete anos de conclusão de curso nas figuras 6 e 8 . Caso fossem considerados os mesmos intervalos de sete anos na figura 7 o último intervalo deveria ser 1985-1991 e se perderia informação sobre dados relevantes mais recentes. Note que a figura 7 não pode ser correlacionada facilmente com as 6 e 8, dado que cada ano de conclusão de curso pode incluir estudantes ingressos em diversos anos (essa figura deve ser considerado como um indicador independente de eficiência na formação graduada de "futuros" pesquisadores).

No primeiro intervalo da figura 7, o percentual de graduados em menos que 6 anos é superior ao dos períodos subsequentes. Isto, juntamente com o histórico na seção II, sugere simplesmente que a conclusão do curso era mais fácil. Contudo, houve um fortalecimento qualitativo na formação, evidenciado pela análise dos outros percentuais. Estes mostram um aumento que privilegia menores tempos para a qualificação pós-graduada. Isto vai ao encontro da política das agências de fomento à pesquisa, ciência e tecnologia, e também das atuais exigências do mercado de trabalho, que não permitem mais os grandes tempos de qualificação do passado.

$\mathrm{Na}$ figura 7, todos os ex-alunos do último intervalo (e parcialmente do penúltimo) experimentaram a reforma de 1984. É particularmente curioso que no último intervalo o correspondente percentual de mestres seja superior ao de graduados. Isso ocorre pois, para alguns, o excesso de tempo gasto na graduação foi compensado pelo curto tempo utilizado para a obtenção da qualificação de mestre. Tal resultado pode ser encontrado em outros cursos de ciências exatas e não constitui em si um forte indício de melhoria na formação. Pode, de fato, ser um indício da "massificação" da formação de mestres. Entretanto essa especulação não se aplica ao percentual de doutores formados em menos que 14 anos, no último intervalo, que é, este sim, um forte indicador do grande progresso na direção de uma formação de excelência.

Pode-se, ainda, comparar esse resultado com o crescimento do número de doutores formados pelo $\mathrm{ON}$ e pelo IAG no passado (particularmente o IAG é responsável pela formação do maior número de doutores em atividade no país [5]). No artigo de Maciel [5] pode-se verificar que o ano de 1987 marca o início do aumento significativo de qualificações de doutorado, tanto no IAG quanto no ON. Pode-se estimar (de forma otimista) um intervalo de tempo mínimo de cerca de 10 anos entre a defesa de tese de doutorado e o ingresso na graduação (4 de graduacao, 2 de mestrado, 4 de doutorado). Ou seja, para as duas instituições citadas, o início do crescimento significativo de doutores 


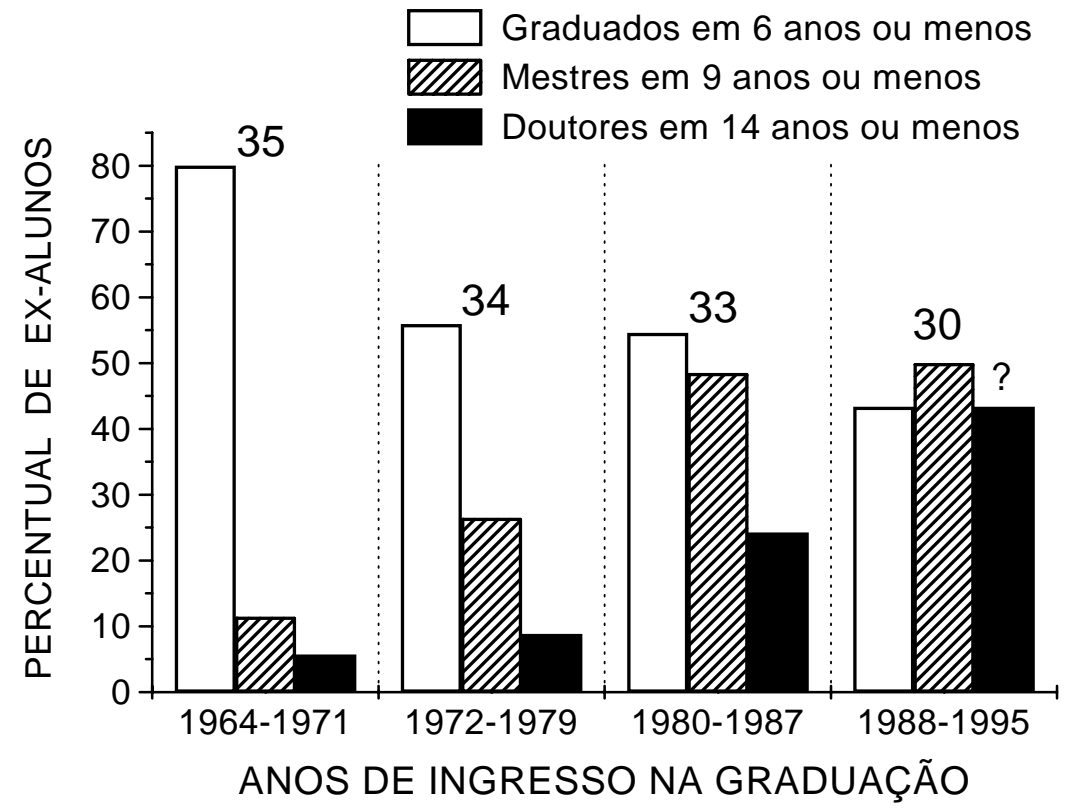

Figura 7. Percentual de alunos que ingressaram no curso e que concluíram a graduação, o mestrado e o doutorado em tempos menores do que, respectivamente, 6, 9 e 14 anos. Os dados foram acumulados e os percentuais calculados em intervalos de oito anos de ingresso no curso. Os tempos de qualificação referem-se, às diferenças entre o ingresso na graduação e, respectivamente: a apresentação de Projeto Final, a dissertação de mestrado e a defesa de tese de doutorado. O número total de alunos ingressos (que concluíram a graduação) em cada intervalo de oito anos está indicado sobre as colunas do respectivo intervalo.

deve-se a alunos ingressos nas graduações em 1977 ou menos, e graduados em 1980 ou menos. Comparando isto com os resultados desse trabalho (melhoria da qualificação de doutorado para alunos ingressos a partir de 1984 e graduados a partir de 1987), concluímos que há uma defasagem de quase uma década entre o início da melhoria significativa de qualificação dos profissionais da comunidade astronômica em geral e de ex-alunos do OV. Isso reforça mais ainda as conclusões acerca do maciço investimento no ensino ocorrido no OV (anterior mesmo à própria melhoria da qualificação do seu quadro), a fim de adequar seus graduados à realidade do progresso nacional em Astronomia.

Nas figuras 6, ou 8, nota-se que o último intervalo contém um número total de ex-alunos menor que os intervalos imediatamente anteriores. Em princípio, isso poderia ser considerado preocupante. Entretanto, observando a figura 5, na qual o intervalo 1995-2002 contém um número relativamente alto de formados, é possível fazer um prognóstico otimista para futuras avaliações. Ou seja, espera-se um aumento de ex-alunos com qualificação pós-graduada.

A figura 8 mostra o percentual de aproveitamento no mercado de trabalho, em intervalos de sete anos de conclusão de curso: foram computados contratos atuais e passados em Astronomia, Física ou Matemática, nos setores acadêmico, técnico e de divulgação/ensino; também foram considerados os percentuais de ex-alunos em atividades de pós-doutorado em Astronomia. No momento, o único setor técnico que contrata astrônomos está na EMBRATEL.

O intervalo 1967-1980 caracteriza-se pela implementação e consolidação do OV e do ON, o que justifica parcialmente o percentual inicial maior de contratos nas áreas acadêmicas da figura 8, pois um percentual significativo de ex-alunos foi absorvido por essas instituições. Tal trajetória de ex-alunos justifica, na figura 6 , os altos percentuais relativos, respectivamente, de mestres em Física ou Matemática no intervalo 1967-1973, e de doutores no intervalo 1974-1980. Em ambos os casos, os contratados foram incentivados pelas exigências da academia. No primeiro intervalo da figura 6, os mestres em Física ou Matemática são, principalmente, ex-professores do OV (à época bastante isolado da comunidade científica) que, sem muitas opções de pós-graduação em Astronomia no país (seção I) e tendo que cumprir grande carga didática docente, não obtiveram o doutorado. Surpreendentemente, os primeiros professores do OV doutores em Astronomia/Astrofísica surgiram somente em meados da década de 1990 (o que subentende um importante autodidatismo entre os docentes do OV, no passado, e uma importante aposta no crescimento do outro, o que pode ser deduzido a partir do sucesso da formação de seus estudantes). Entretanto atualmente, no OV, cerca de 64\% dos docentes têm doutorado em Astronomia.

Nota-se, na figura 8, o decréscimo dos percentuais para as instituições de pesquisa e ensino de Astronomia no intervalo 1967-1987. Porém, somando-se o percentual de contratos e de pós-doutorados no último intervalo da figura, e considerando o grande desenvolvimento e expansão da Astronomia no Brasil, é de se esperar um aumento futuro significativo dos percentuais de contratações em instituições que realizam pesquisa e ensino de Astronomia e Astrofísica.

Finalmente, vale ressaltar que, apesar das peculiaridades das evoluções das áreas e instituições astronômicas, ao longo de 20 anos o índice de aproveitamento de ex-alunos do 
curso da UFRJ, em Astronomia e áreas correlatas, mantevese, em média, acima de $60 \%$, com a tendência de aumento evidenciada na figura 8 .

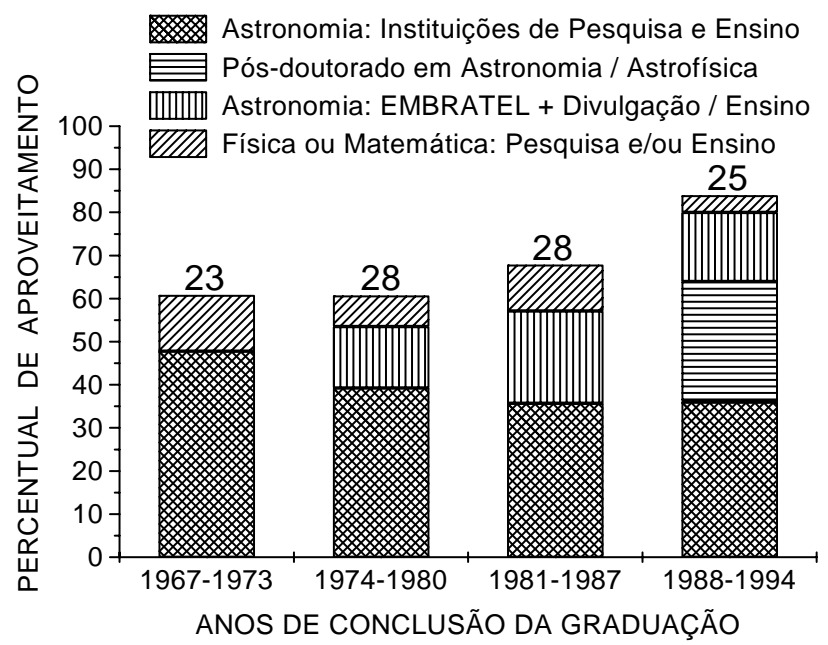

Figura 8. Percentual de alunos aproveitados no mercado de trabalho, ou realizando pós-doutorados, em relação a alunos que concluíram a graduação. Os dados foram acumulados e os percentuais calculados em intervalos de sete anos de conclusão do curso. $\mathrm{O}$ número total de alunos que concluíram a agraduação em cada intervalo de sete anos está indicado sobre a coluna do respectivo intervalo ( $c f$. figura 6).

\section{Observações e Conclusões Finais}

Nesse trabalho mostramos as conseqüências do crescimento do Observatório do Valongo/UFRJ e da melhoria no ensino sobre a formação discente.

Um processo global de avaliação requerido pela UFRJ veio ao encontro das discussões iniciadas no $\mathrm{OV}$, a partir de meados da década de 1990, com o objetivo de realizar uma grande reforma curricular (que está sendo implantada). Nesse contexto iniciou-se o estudo de caso, a fim de encontrar indicadores de avaliação de eficiência da formação do graduado em Astronomia. A análise de erros e acertos também norteou a discussão de reforma curricular e pode igualmente ser utilizada em futuros levantamentos de desempenho.

As metas para o curso de graduação de Astronomia da UFRJ são o aperfeiçoamento e a modernização da formação dos alunos e o fortalecimento da iniciação científica. Tratando-se de graduação, o objetivo de seu currículo deve ser o de permitir ao aluno estar apto a alternativas de sobrevivência. Assim, a este nível, a formação deve ser básica, geral e atualizada o suficiente para que o aluno possa ser inserido no mercado, ou seguir a carreira acadêmica, sem grandes problemas.

Nesse trabalho mostrou-se que o percentual, relativamente alto, de aproveitamento de ex-alunos em Astronomia e áreas correlatas, formados ao longo de 20 anos, mantevese razoavelmente constante e tende a aumentar (figura 8). Essa expectativa baseia-se no grande desenvolvimento da
Astronomia Brasileira na pesquisa científica, com reflexos também no crescente interesse na implantação de atividades de graduação e nas atividades da divulgação científica de qualidade, junto ao público em geral e junto ao ensino médio e fundamental.

Exemplos das atividades de divulgação da Astronomia, que receberam verbas do $\mathrm{CNPq}$ (edital de educação de 2001), são: i) a Olimpíada Brasileira de Astronomia que passou a ser organizada pela SAB [14], atingindo milhares de alunos em todo o Brasil, e que realizou sua quinta edição, sob a coordenação de Jaime F. Villas da Rocha (UERJ); ii) o Projeto ENSINAST, coordenado por Horácio A. Dottori (UFRGS) e Adriana V. R. da Silva (CRAAM/Mackenzie), envolvendo 15 instituições brasileiras (o OV incluído).

O grande sucesso demonstrado pela diminuição do tempo para a qualificação de pós-graduação dos ex-alunos que experimentaram a reforma curricular de 1984 (e outras melhorias posteriores implementadas no OV, segundo as seções I e II), assim como a participação expressiva de ex-alunos no mercado de trabalho da comunidade astronômica brasileira, deixa claro que a modernização curricular periódica é uma grande necessidade em qualquer área na qual as revoluções técnicas provocam rápidas transformações de paradigma. Entretanto, devido ao histórico apresentado nesse estudo, não podemos ignorar a relevância, para o sucesso na formação graduada, do acompanhamento acadêmico "eticamente vivenciado" (seção II) que torna o ambiente acadêmico mais atrativo ao estudante e que no caso do OV seguramente salvou algumas carreiras.

Os indicadores aqui apresentados claramente refletem a melhoria na formação dos alunos, mas também apontam algumas falhas, tais como o aumento médio do tempo de permanência para a conclusão do curso e a diminuição de alunos formados entre os ingressos no intervalo 1964-1995 (figura 7). Além disso, desde 1992 houve um aumento do número de vagas oferecidas anualmente, de 20 para 30, mas tal medida não teve uma influência direta sobre o número de alunos formados.

Espera-se, com a nova reforma curricular, diminuir a taxa de evasão e o prazo de integralização e esperase aumentar a participação dos estudantes de graduação nas atividades acadêmicas (esse aumento deve ser estimulado também pela implantação da pós-graduação no $\mathrm{OV}$ - seção I). Objetiva-se atingir a excelência através da sólida formação nas disciplinas de Física e nas técnicas de observação e de redução de dados da moderna Astronomia. Baseadas nas discussões curriculares, e no amadurecimento da insitutição, várias melhorias têm sido implementadas e já apresentam reflexos, tal como o aumento do número de alunos formados nos últimos anos (ver figuras 2 e 5 ).

Foi enfatizado que o OV reúne as características de ter um curso científico, inserido em uma sociedade jovem (SAB). Além dos grandes avanços tecnológicos que mudaram a face da Astronomia, houve uma rápida evolução no OV (figura 1). Tais circunstâncias permitem um diagnóstico de trajetória institucional para a adequação da estrutura de curso às diferentes necessidades da carreira científica. 
Infelizmente, devido (até onde se sabe) ao ineditismo de tal análise, não é possivel comparar os resultados com diagnósticos análogos para outros cursos de graduação. Tomando por base a evolução da qualidade da formação discente no curso do OV, pode-se sugerir como metas de uma boa formação graduada cerca de $60 \%$ de qualificação de doutorado entre ex-alunos (com tempos pequenos para a qualificação, figuras 6 e 7) e cerca de $70 \%$ de ex-alunos absorvidos no mercado de trabalho em áreas correlatas ao da graduação (figura 8). Certamente tal perspectiva leva em conta a adequação dos projetos nacionais para a ciência e tecnologia à perspectiva de crescimento nas áreas científicas.

Um dos principais resultados desse trabalho foi a criação dos indicadores de longo prazo apresentados na seção IV. A figura 7 mostra que, no intervalo 1964-1995, apesar de ter aumentado o pecentual de alunos formados em mais do que 6 anos e de ter diminuído a média de alunos formados, a qualidade da formação aumentou ( $c f$. figuras 6 a 8). Portanto, os indicadores mostram que os índices de diplomação, por si só, não são suficientes (e podem ser inadequados) para a avaliação da formação científica.

Os levantamentos e análises aqui apresentados exigiram grande empenho e parcialmente só foi possível realizar tal trabalho devido ao número relativamente pequeno de alunos. Entretanto, com recursos computacionais ora disponíveis para armazenamento de dados e troca de informação, certamente será viável a execução de futuros levantamentos e análises, mesmo para cursos com um número elevado de estudantes. Acredito que este trabalho possa indicar os tipos de dados potencialmente úteis para futuros indicadores de eficiência na formação e de aproveitamento vocacional de graduados em ciências exatas.

\section{Agradecimentos}

Agradeço aos Professores do OV: Encarnaciòn Gonzalez e José Adolfo de Campos (responsáveis pelo início do levantamento de dados de ex-alunos), Gustavo Porto de Mello, Heloisa Boechat-Roberty, Sueli Guillens e Jorge Albuquerque Vieira, e ao pós-doutor da UERJ Jaime Rocha, pelas dis- cussões ao longo dos anos sobre temas aqui abordados, por revisões e sugestões; ao árbitro que contribuiu para tornar algumas partes mais compreensíveis; aos (muitos outros) não citados que transformaram um evento improvável em uma história que pode ser contada. À FUJB e ao OV pelo apoio computacional.

\section{Referências}

[1] COOPERA (Comissão Permanente de Avaliação), Instituto de Geociências, UFRJ, $1^{a}$ edição (1995)

[2] Arany-Prado, L.I., Boletim da SAB 17, nº 2, 23-44 (1998)

[3] de Campos, J.A.S., em: A Astronomia no Brasil: Depoimentos, eds.: B. Barbuy, J. Braga, N. Leister, SAB, IAG/USP, 93-105 (1994)

[4] Bretones, P.S., Compiani, M., Boletim da SAB 20, n ${ }^{o} 3$, 61$82(2001)$

[5] Maciel, W.J., Boletim da SAB 16, nº 2, pg. 11-31 (1996)

[6] da Silva, L., em: A Astronomia no Brasil: Depoimentos, eds.: B. Barbuy, J. Braga, N. Leister, SAB, IAG/USP, 83-92 (1994)

[7] Arany-Prado, L.I., Boletim da SAB 20 , n ${ }^{o}$ 3, 83-90 (2001)

[8] Arany-Prado, L.I., In: VIII Encontro de Pesquisa em Ensino de Física, SBF (2002)

[9] COOPERA, Avaliação Institucional do Centro de Ciências Matemáticas e da Natureza (CCMN)/UFRJ - Relatório Final, 108-113 (1997)

[10] Barbuy, B., Lapasset. E., Baptista, R., Cid Fernandes, R., (eds.), Proceedings of the Workshop Science with Gemini, IAG/USP \& UFSC (1998)

[11] Notícias do boletim da SAB 20, n $^{\circ} 2$ (2000)

[12] Guimarães, J.A., Jornal da Ciência de novembro, SBPC (1999)

[13] Benevides-Soares, P., Boletim da SAB 19, $\mathrm{n}^{\circ}$ 2, 61-70 (1999)

[14] Canalle, J.B.G., Lavouras, D.F., Arany-Prado, L.I., Oliveira Abans, M., Boletim da SAB 20, $\mathrm{n}^{o}$ 2, 57-71 (2000) 